\title{
Tunneling Spectroscopy by Nuclear Magnetic Resonance: Analysis of Rotational Tunneling in Solid Pentamethylbenzene
}

\author{
W. T. Sobol *, K.R. Sridharan, I. G. Cameron, and M. M. Pintar \\ Guelph-Waterloo Program for Graduate Work in Physics, \\ Waterloo Campus, University of Waterloo, Waterloo, Ontario, Canada N2L 3G1.
}

Z. Naturforsch. 40a, 1075-1084 (1985); received August 10, 1985

\begin{abstract}
The frequency dependence of the spin-lattice relaxation time $T_{1}$ was measured at three temperatures near one of the Zeeman-tunneling level matching resonances for pentamethylbenzene. These measurements are correlated with $T_{1}$ temperature dependence data from the literature. It is shown that the frequency dependence of the Zeeman-torsion coupling time cannot be explained in terms of the semiclassical perturbation theory using time correlation functions. A three bath polarization transfer model is also employed and the applicability of both models discussed. Zeeman-torsion coupling is further investigated using a saturation sequence and the results are compared with the predictions of the three bath polarization transfer model.
\end{abstract}

\section{Introduction}

The effect of rotational tunneling on the proton spin-lattice relaxation of $\mathrm{CH}_{3}$ torsional oscillators embedded in a solid matrix has been studied extensively. The initial theoretical models $[1,2]$ have been modified to fit the extensive experimental data. These data are mainly temperature dependences of the proton spin-lattice relaxation time for lattices with very different hindering barriers. The theoretical approaches presently used are based on the BPP perturbation calculation [3-7], where it is assumed that the same random process governs all the relaxation processes in the sample. The resulting equation for the relaxation rate (which is identified with the initial slope of the relaxation decay) contains spectral densities of the form

$$
\begin{array}{r}
J\left(k \omega_{\mathrm{t}} \pm n \omega_{0}\right) \propto \frac{\tau}{1+\left(k \omega_{\mathrm{t}} \pm n \omega_{0}\right)^{2} \tau^{2}}, \\
\text { with } \quad k=0,1 ; n=1,2,
\end{array}
$$

where $\omega_{0}$ is the frequency of the resonant r.f. field, $\omega_{\mathrm{t}}$ the tunneling frequency, and $\tau$ the correlation time of the random motion which causes the relaxation.

This model tas developed to explain the experimental temperature dependence of the relaxation time $T_{1}$. Since $\tau$ and $\omega_{\mathrm{t}}$ are both temperature de-

\footnotetext{
* On leave from the Silesian University, Katowice, Poland. Reprint requests to Prof. M. M. Pintar, Waterloo Campus, University of Waterloo, Waterloo, Ontario, Canada N2L 3Gl.
}

pendent, the following relations have been suggested to account for the temperature dependence [6]:

$$
\begin{aligned}
\frac{1}{\tau}= & \frac{1}{\tau_{0}} \exp \left\{-E_{\mathrm{a}} / R T\right\} \\
& +\sum_{i} \frac{1}{\tau_{\mathrm{t} i}} \exp \left\{-E_{0 i} / R T\right\}, \\
\omega_{\mathrm{t}}= & \frac{\omega_{\mathrm{t} 0}+\sum_{i} \omega_{\mathrm{t} i} \exp \left\{E_{0 i} / R T\right\}}{\sum_{i} \exp \left\{E_{0 i} / R T\right\}},
\end{aligned}
$$

where $R$ is the universal gas constant, $E_{0 i}$ the energy difference between the ground torsional state and the $i$-th excited torsional state, $\omega_{\mathrm{t} i}$ the tunneling splitting of the $i$-th torsional level (in $\mathrm{rad} \mathrm{sec}^{-1}$ units), $E_{\mathrm{a}}$ the activation energy (in the classical sense) of thermal random motion and $T$ the temperature. The parameters $\tau_{0}$ and $\tau_{\mathrm{t} i}$ are phenomenological constants in the assumed Arrhenius-type temperature dependence.

From (1) and (2) it follows that in the development of the tunneling assisted relaxation theory (TART) is was assumed implicitly that the couplings between different reservoirs (Zeeman, tunneling and the lattice) are affected by the relaxation processes in exactly the same manner, so that the same "effective" correlation time $\tau$ enters all spectral densities. In other words, the lattice (phonons) always supplies or absorbs the necessary amount of energy to compensate for any mismatch of the energy levels involved. 
The TART approach has been very successful. It quantitatively describes the temperature dependence of $T_{1}$ for samples with very low hindering barriers (i.e. satisfying the condition $\omega_{\mathrm{t}}(T) \gg \omega_{0}$ ). In particular, it explains the presence of an additional extremum (caused by the condition $\omega_{\mathrm{t}} \tau \approx 1$ ) on the relaxation curve as well as the different "apparent" activation energies on the high and low temperature sides of the relaxation peak [6]. For samples with higher hindering barriers, such that at some temperature the "level matching" condition $\omega_{\mathrm{t}}=n \omega_{0}$ can occur, it quantitatively describes the experimental values of $T_{1}$, as long as the experiments are performed at frequencies far from the resonant matching conditions $\omega_{\mathrm{t}}=\omega_{0}$ and $\omega_{\mathrm{t}}=$ $2 \omega_{0}$. It also correctly predicts the presence of additional "resonance" extrema in the $T_{1}$ temperature dependence when the $\omega_{\mathrm{t}}=\omega_{0}$ or the $\omega_{\mathrm{t}}=2 \omega_{0}$ level matching occurs. However, there is a very significant consistent discrepancy between the measured and modelled values of $T_{1}$ at the matching frequencies $[4,7-9]$. It is very difficult to identify the source of this problem from the temperature dependence data alone. In the spectral densities, (1), both $\tau$ and $\omega_{\mathrm{t}}$ are temperature dependent, so that near a level-matching condition both influence the proton spin relaxation rate. An insight into these problems can be obtained by studying the frequency dependence of $T_{1}^{-1}$ at several chosen temperatures. Moreover, in the level-matching region the relaxation is usually distinctly nonexponential. The study of all relaxation components, instead of the initial slope only, also provides additional information, in particular about the validity of (2), since these components depend differently on different couplings between various energy reservoirs.

Finally, one can perform a so-called "population transfer" experiment, by applying a saturation sequence of $\pi / 2$ pulses, separated by a time $\tau$, to the sample when the frequency satisfies a level-matching condition [10]. It is very difficult to explain the results of this type of experiment in terms of TART, since the initial slope is hardly affected by the change in the state of the tunneling system. However, the data provide important new information about the specific heats of the reservoirs involved.

In order to understand the source of the discrepancies between the experimental data and TART we performed a set of spectroscopic experiments (frequency dependence of $T_{1}$ at a few selected tem- peratures), as well as saturation experiments in the level-matching frequency range with a sample for which the temperature dependance of $T_{1}$ has been known. We have chosen pentamethylbenzene (PMB) since in the literature several temperature dependences of the spin-lattice relaxation time at several frequencies [11-16] have been reported. In addition, published experimental data indicated that one of the level-matching conditions $\left(\omega_{\mathrm{t}}=2 \omega_{0}\right)$ falls within the range of frequencies, $5-65 \mathrm{MHz}$, which are experimentally accessible in our laboratory.

We have used TART to model the known temperature dependencies as accurately as possible (which has not been attempted before), and compare the obtained parameters with our frequency dependent results as well as with the results of the resonant population transfer experiments. In this way we were in a position to identify the discrepancy between the predictions of the TART model and the experiment in the level-matching regions rather accurately. On the basis of the published experimental results and the present experimental evidence, in particular in the Zeeman-torsional resonant range of frequencies, it was possible to identify the origin of the failure of the correlation approach to deal with the resonant Zeeman-torsional transitions.

\section{Experimental}

The $99 \%$ pure polycrystalline pentamethylbenzene (PMB) used in this research was purchased from the Aldrich Chemical Company. It was crushed and the powder sealed under vacuum in an $8 \mathrm{~mm}$ O.D. glass tube.

The $T_{1}$ temperature dependences presented in this paper were obtained from the literature $[13,15,16$, 17]. These measurements were performed using standard techniques. At $60 \mathrm{MHz}$ some of the recovery functions were nonexponential and, where possible, were characterized by a fast and a slow time constant $[15,16]$. At all other frequencies the recovery functions were exponential.

These temperature dependences were supplemented by $T_{1}$ frequency dependences which were obtained using a Bruker SXP variable frequency spectrometer and a Varian 15" electromagnet. These experiments were performed using a $\pi / 2-\tau-\pi / 2$ 
pulse sequence. The length of the $\pi / 2$ pulse was around $3 \mu \mathrm{s}$ and the deadtime ranged from about $4 \mu \mathrm{s}$ at the highest frequencies to about $10 \mu \mathrm{s}$ at lower frequencies. The signal to noise ratio varied from $30: 1$ to $150: 1$ depending on the temperature and frequency of the experiment. When the signal to noise ratio was low, several acquisitions of the free induction decay (FID) were taken and averaged so that in all cases the effective signal to noise ratio was about $100: 1$ or better. The NMR signal was phase detected and fed into a Biomation 805 analog to digital converter, which was on-line to a Hewlett Packard 9845 A computer. The signal was recorded at about 20 different time windows on the FID. The magnetization value at each of these windows was obtained by averaging over $2 \mu$ s of the FID.

A saturation sequence consisting of a train of four $\pi / 2$ pulses separated by $\tau, \pi / 2^{-}[\tau-\pi / 2]_{3}$, was also applied to the sample using a standard Bruker pulse programmer. This sequence was used to saturate the magnetization selectively [18]. The amplitude $M_{n}$ of the FID recorded at the end of each pulse was measured for various pulse spacings $\tau$.

The sample, which was housed in a Janis 5 RD liquid helium cryostat, was kept at a constant temperature by balancing the rate of flow of cold helium gas past the sample with the current through a heater which surrounds the sample container. With this technique it was possible to keep the sample temperature stable to within $\pm 0.1 \mathrm{~K}$. For temperatures above $30 \mathrm{~K}$ the sample temperature was monitored by a platinum sensor placed near the sample, whereas for temperatures below $30 \mathrm{~K}$ a germanium sensor was used. The sample was cooled from room temperature to the desired temperature in about two hours.

The probe and sample holder were home built and the matching scheme was based on a design by Clark [19].

\section{Results}

From published data it is known that PMB has two solid phases with the phase transition occurring at $297 \mathrm{~K}$ [11]. In the low temperature phase there are three non-equivalent types of methyl groups, and their ground state tunneling splittings are $\omega_{\mathrm{t}}^{A} /$ $2 \pi \sim 1 \mathrm{MHz}[13,16,19], \omega_{\mathrm{t}}^{B} / 2 \pi=160 \pm 8 \mathrm{MHz}[8$,

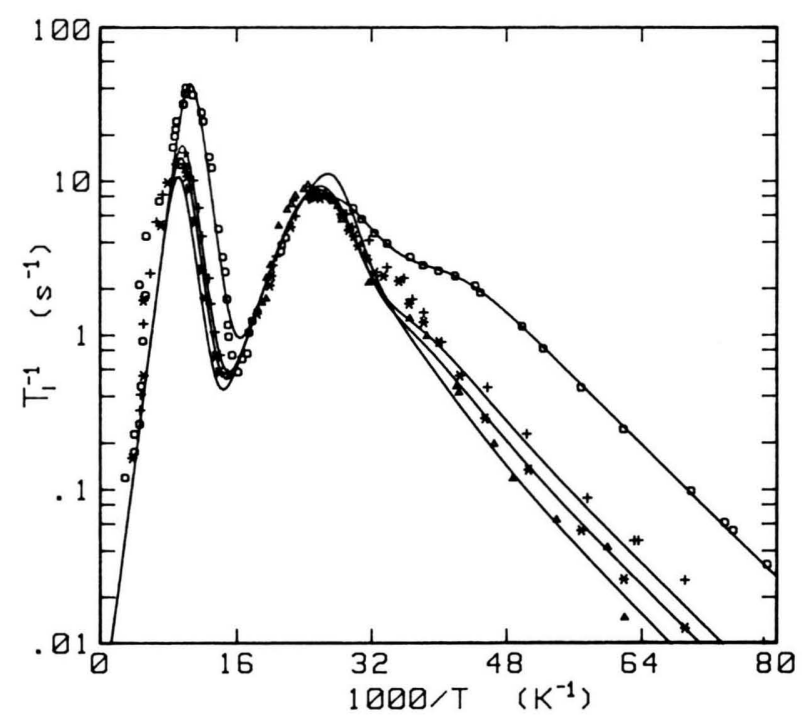

Fig. 1. The temperature dependences at 10, 25, 30 and $40 \mathrm{MHz}$ are indicated by circles, crosses, asterisks and triangles, respectively. The theoretical curves were computed using all of the spectral densities in (4) and the parameters given in Table 2 . At $\beta \approx 30 \mathrm{~K}^{-1}$ and $40 \mathrm{MHz}$ the effect of the resonant spectral density $J\left(2 \omega_{0}-\omega_{\mathrm{t}}\right)$ begins to show up.

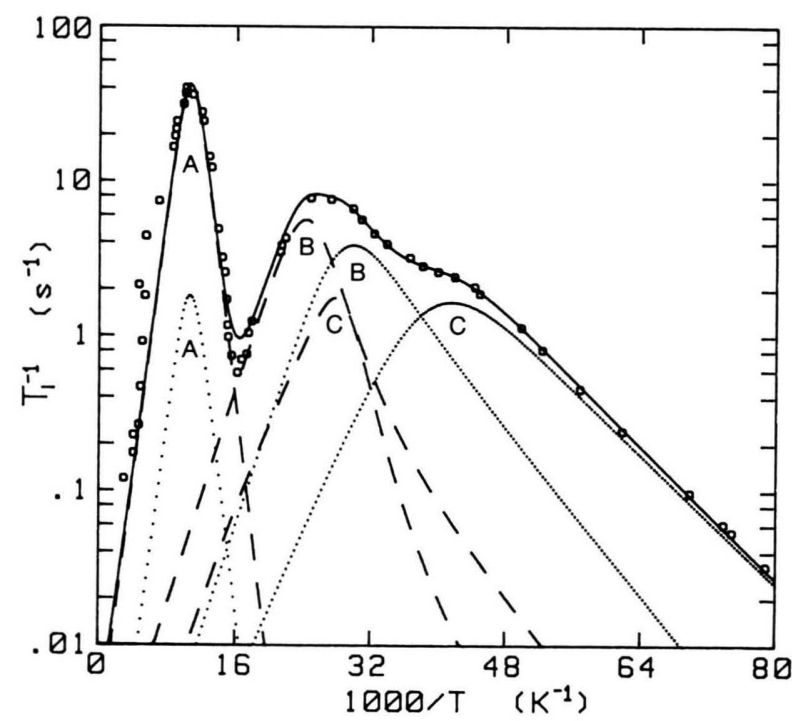

Fig. 2. The temperature dependence at $10 \mathrm{MHz}$ indicating the various groups of spectral densities and where they contribute. The solid line is the total $T_{1}^{-1}$ value. The dotted lines correspond to the spectral densities $J\left(\omega_{0}\right)+J\left(2 \omega_{0}\right)$ while the dashed lines represent the contributions due to $J\left(n \omega_{0} \pm \omega_{\mathrm{t}}\right)$ for $n=1$ and 2 . The letters A, B and C refer to the three non-equivalent methyl groups as described in the text. 


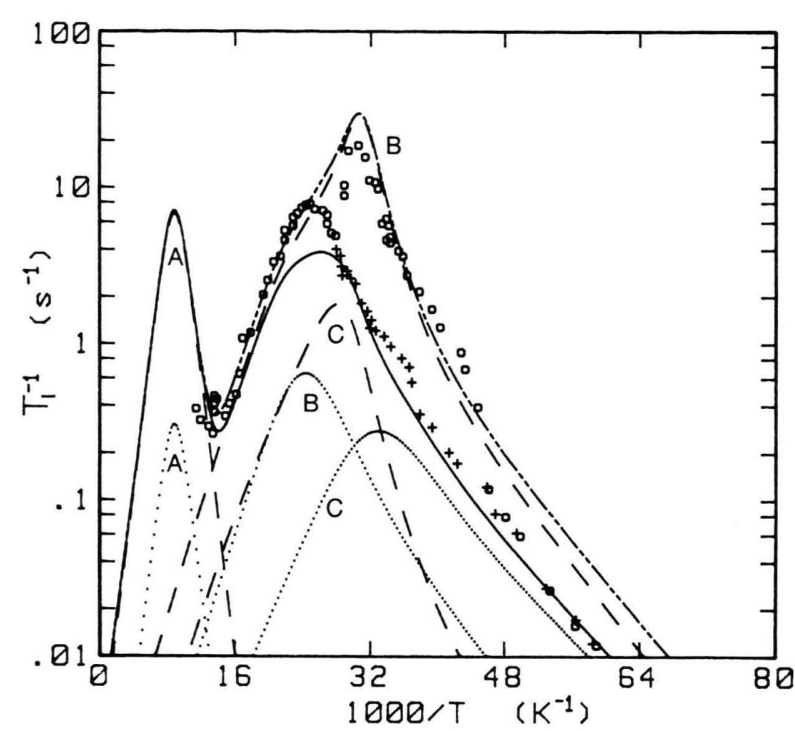

Fig. 3. The temperature dependence at $60 \mathrm{MHz}$. The circles represent initial slope values and the crosses represent the long time constant when the recovery functions were decomposed. The dotted lines correspond to the spectral densities $J\left(n \omega_{0}\right)$ for $n=1$ and 2 while the dashed lines represent the contributions due to $J\left(n \omega_{0} \pm \omega_{\mathrm{t}}\right)$ for $n=1$ and 2. The "dot-dot-dash" line represents the full $T_{1}^{-1}$ value with all spectral densities considered and the solid curve corresponds to the full $T_{1}^{-1}$ value minus the resonant spectral density $J\left(2 \omega_{0}-\omega_{t}\right)$. The letters A, B and C refer to the three non-equivalent methyl groups as described in the text.

9], $\omega_{\mathrm{t}}^{C} / 2 \pi=1.5 \pm 0.1 \mathrm{GHz}$ [20]. Their relative population ratios were estimated to be $2: 2: 1$ [20]. From the temperature dependence of $T_{1}$ (Figs. $1-3)$ it is apparent that in the low temperature phase there are three $T_{1}$ minima $[13,15,16,17]$. The high temperature minimum, which occurs at about $114 \mathrm{~K}$, follows the classical BPP type behavior but the $T_{1}$ minimum at $40 \mathrm{~K}$ is independent of frequency. The $T_{1}$ minimum at about $25 \mathrm{~K}$, while it does show a frequency dependence, cannot be explained using the BPP modelling. Furthermore, since it is not well resolved, the quantitative analysis of this minimum was never completed. These findings were also supported by $T_{1 \varrho}$ and $T_{1 D}$ experiments $[13,17]$.

At $60 \mathrm{MHz}$ another clearly resolved $T_{1}$ minimum, which was not seen at lower frequencies, has been observed at $32.2 \mathrm{~K}[15,16]$. This minimum, which coincides with appreciable nonexponentiality in the magnetization recovery, was attributed to the level matching condition $\omega_{\mathrm{t}}=2 \omega_{0}$. From this informa- tion, along with the usual form of the tunneling splitting temperature dependence, it was concluded that at $32 \mathrm{~K}$ the tunneling splitting $\omega_{\mathrm{t}}^{B} / 2 \pi$ has decreased to about $120 \mathrm{MHz}$. From this observation it has been deduced that the neighbouring minimum at $40 \mathrm{~K}$ is due to the $\omega_{0}=\omega_{\mathrm{t}}^{B}$ resonance. This means that at $40 \mathrm{~K}$ the tunneling frequency $\omega_{\mathrm{t}}^{B} / 2 \pi$ has decreased to $60 \mathrm{MHz}$.

The $T_{1}$ frequency dependences reported in this paper were performed at 25,32 and $40 \mathrm{~K}$. The relaxation was apparently exponential between 20 and $40 \mathrm{MHz}$ at all three temperatures but above $40 \mathrm{MHz}$ it became nonexponential. In this higher frequency region the $T_{1}$ decay was fit to the sum of two exponentials at all windows on the FID. Thus all of the high frequency $T_{1}$ decays were characterized by a relaxation rate and a magnetization fraction for each of these two components. The accuracy of any of the relaxation rates measured was better than $10 \%$. We shall designate the larger $T_{1}$ value as $T_{1 L}$ and the smaller $T_{1}$ value as $T_{1 S} . T_{1 L}$ follows the classical BPP behavior and agrees with the data in the literature $[11-16] . T_{1 S}$, however, has a strong frequency dependence which exhibits a "resonance-like" maximum. The magnetization component associated with the long relaxation time accounted for about $75 \%$ of the total magnetization. Since there was very good agreement at all windows for each relaxation time, the final values were obtained by averaging over all windows.

The results of the relaxation rate measurements at $25 \mathrm{~K}$ are presented in Figure 4 . In the frequency range $22-43 \mathrm{MHz}$ the relaxation is apparently exponential. Two components were barely distinguishable in the range $43-50 \mathrm{MHz}$ whereas at frequencies higher than $50 \mathrm{MHz}$ two components were clearly resolved. $T_{1 S}^{-1}$ increases monotonically with frequency up to the upper limit of the frequency range of our apparatus. $T_{1 L}^{-1}$ at this temperature is almost frequency independent.

The results of measurements at $32 \mathrm{~K}$ are presented in Figure 5. These experiments were performed in the frequency range $48-65 \mathrm{MHz} . T_{1 S}^{-1}$ has an extremum $\left(T_{1 S, \min }=26 \mathrm{~m} \mathrm{sec}\right)$ at the frequency $62 \mathrm{MHz} . T_{1 L}^{-1}$ is almost constant in the frequency range studied.

At $40 \mathrm{~K}$ measurements were performed in the frequency range 14 to $63.5 \mathrm{MHz}$ and the results are shown in Figure 6. For frequencies between 44 and $63 \mathrm{MHz}$ the observed magnetization recovery was 


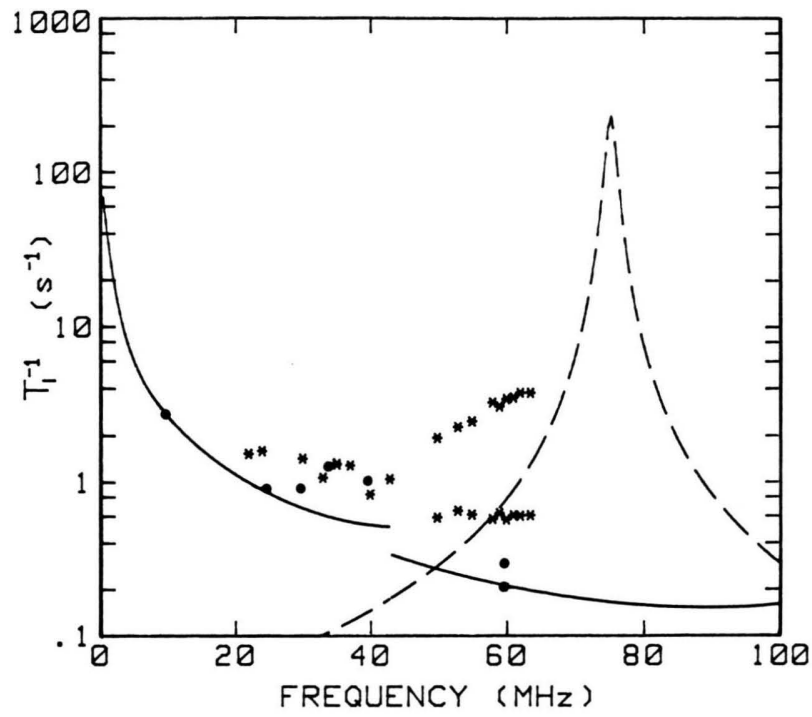

Fig. 4. The frequency dependence at $25 \mathrm{~K}$. The dots represent values taken from the literature while the asterisks correspond to data obtained in our lab. In the region where only one time constant is reported the $T_{1}^{-1}$ value was determined by using all of the spectral densities. In the region where two time constants were used the long component was fit using all but the resonant spectral density (solid line) and the short component was fit using only the resonant term (dashed line).

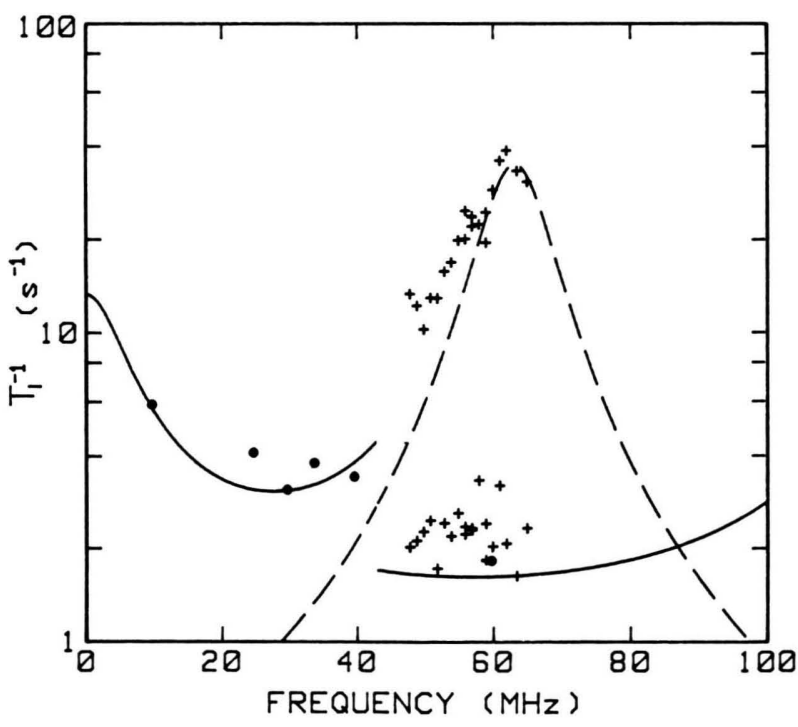

Fig. 5. The frequency dependence at $32 \mathrm{~K}$. The dots represent values taken from the literature while the crosses correspond to data obtained in our lab. The meaning of the lines is the same as in Figure 4.

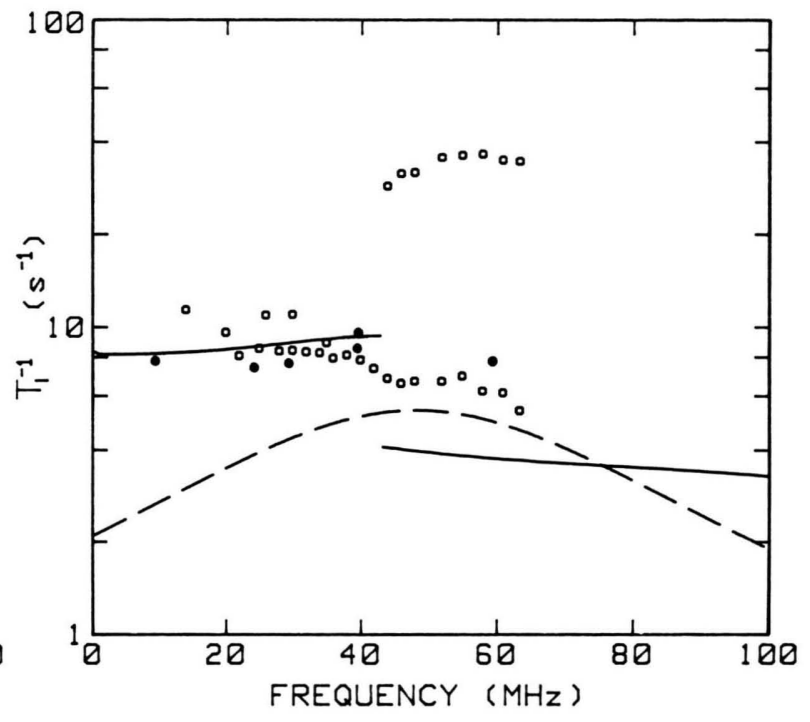

Fig. 6. The frequency dependence at $40 \mathrm{~K}$. The dots represent values taken from the literature while the circles correspond to data obtained in our lab. The meaning of the lines is the same as in Figure 4.

decomposed into two exponential recoveries. $T_{1 S}^{-1}$ again exhibits a resonance-like behavior but, in this case, it is not as sharp as it was at $32 \mathrm{~K}$. The extremum of this relaxation time $\left(T_{1 S, \min }=27 \mathrm{~ms}\right)$ was found at $58 \mathrm{MHz} . T_{1 L}^{-1}$ decreases with frequency in the whole range of observation.

The results of a four-pulse saturation experiment, performed at $32 \mathrm{~K}$ and $62 \mathrm{MHz}$, for various mixing times $\tau$ are presented in Table 1. At this frequency, which corresponds to the maximum in the $T_{1 S}^{-1}$ resonant peak, $T_{1 S}$ is $26 \mathrm{~ms}$ and $T_{1 L}$ is $480 \mathrm{~ms}$. It was observed that the magnetization after the $n^{\text {th }}$ pulse is smaller than the magnetization following the $(n-1)^{\text {th }}$ pulse. This is consistent with the results of similar experiments performed on other materials which show that the sub-system contributing the magnetization can be saturated in a time much shorter than $T_{1}$, and for this reason this system must be something other than the lattice $[10,18]$. These results will be discussed in more detail later in terms of the polarization transfer model. 
Table 1. Results of a four $\pi / 2$ pulse saturation experiment on PMB at $32 \mathrm{~K}$ and $62 \mathrm{MHz}$. $\tau$ is the mixing time between pulses. The values of $T_{1 S}$ and $T_{1 L}$ are 26 and $480 \mathrm{~ms}$, respectively.

\begin{tabular}{|c|c|c|c|c|c|c|c|c|c|c|c|}
\hline \multirow{2}{*}{$\begin{array}{l}\tau \\
{[\mathrm{ms}]}\end{array}$} & \multirow{2}{*}{$\frac{\tau}{T_{1 S}}$} & \multicolumn{5}{|c|}{ Experimental } & \multicolumn{5}{|c|}{ Calculated } \\
\hline & & $M_{1} / M_{0}$ & $M_{2} / M_{0}$ & $M_{3} / M_{0}$ & $M_{2} / M_{1}$ & $M_{3} / M_{2}$ & $M_{1} / M_{0}$ & $M_{2} / M_{0}$ & $M_{3} / M_{0}$ & $M_{2} / M_{1}$ & $M_{3} / M_{2}$ \\
\hline 10 & 0.39 & 0.11 & 0.07 & 0.06 & 0.68 & 0.85 & 0.07 & 0.07 & 0.06 & 0.94 & 0.93 \\
\hline 20 & 0.77 & 0.14 & 0.10 & 0.08 & 0.71 & 0.83 & 0.13 & 0.11 & 0.10 & 0.89 & 0.89 \\
\hline 40 & 1.54 & 0.17 & 0.13 & 0.11 & 0.77 & 0.83 & 0.20 & 0.17 & 0.15 & 0.85 & 0.86 \\
\hline 60 & 2.31 & 0.21 & 0.15 & 0.13 & 0.73 & 0.84 & 0.26 & 0.21 & 0.19 & 0.84 & 0.87 \\
\hline 100 & 3.85 & 0.28 & 0.21 & 0.18 & 0.74 & 0.87 & 0.33 & 0.28 & 0.25 & 0.86 & 0.89 \\
\hline 200 & 7.69 & 0.40 & 0.32 & 0.29 & 0.79 & 0.93 & 0.46 & 0.42 & 0.39 & 0.91 & 0.94 \\
\hline
\end{tabular}

Table 2. Numerical values of constants used in modelling.

\begin{tabular}{|c|c|c|c|c|c|c|c|c|c|}
\hline \multirow{2}{*}{$\begin{array}{l}\text { Type of } \\
\mathrm{CH}_{3} \text { group } \\
(j)\end{array}$} & \multicolumn{2}{|c|}{$\begin{array}{l}\text { Dipolar constants } \\
{\left[\mathrm{s}^{-2}\right]}\end{array}$} & \multicolumn{2}{|c|}{$\begin{array}{l}\text { Correlation times } \\
{[\mathrm{s}]}\end{array}$} & \multicolumn{3}{|c|}{$\begin{array}{l}\text { Activation energies } \\
{\left[\mathrm{kcal} \mathrm{mole}^{-1}\right]}\end{array}$} & \multicolumn{2}{|c|}{$\begin{array}{l}\text { Tunneling splittings } \\
{\left[\mathrm{rad} \mathrm{s}^{-1}\right]}\end{array}$} \\
\hline & $C_{\mathrm{EE}}^{j}$ & $C_{\mathrm{AE}}^{j}$ & $\tau_{0}^{j}$ & $\tau_{\mathrm{t} i}^{j}$ & $E_{\mathrm{a}}^{j}$ & $i$ & $E_{0 i}^{j}$ & $k$ & $\omega_{t k}^{j}$ \\
\hline A & $8.0 \times 10^{7}$ & $9.0 \times 10^{8}$ & $2.33 \times 10^{-13}$ & $1.0 \times 10^{-10}$ & 2.02 & $\begin{array}{l}1 \\
2 \\
3\end{array}$ & $\begin{array}{l}1.94 \\
0.00 \\
0.00\end{array}$ & $\begin{array}{l}0 \\
1 \\
2 \\
3\end{array}$ & $\begin{array}{l}6.24 \times 10^{6} \\
4.52 \times 10^{8} \\
0.00 \\
0.00\end{array}$ \\
\hline B & $1.7 \times 10^{8}$ & $6.48 \times 10^{8}$ & $1.2 \times 10^{-13}$ & $2.0 \times 10^{-10}$ & 0.80 & $\begin{array}{l}1 \\
2 \\
3\end{array}$ & $\begin{array}{l}0.31 \\
0.63 \\
1.00\end{array}$ & $\begin{array}{l}0 \\
1 \\
2 \\
3\end{array}$ & $\begin{array}{l}1.00 \times 10^{9} \\
3.00 \times 10^{10} \\
7.00 \times 10^{11} \\
8.40 \times 10^{12}\end{array}$ \\
\hline $\mathrm{C}$ & $7.3 \times 10^{7}$ & $5.77 \times 10^{8}$ & $1.2 \times 10^{-13}$ & $1.0 \times 10^{-10}$ & 0.60 & $\begin{array}{l}1 \\
2 \\
3\end{array}$ & $\begin{array}{l}0.24 \\
0.40 \\
0.75\end{array}$ & $\begin{array}{l}0 \\
1 \\
2 \\
3\end{array}$ & $\begin{array}{l}9.43 \times 10^{9} \\
5.00 \times 10^{11} \\
2.80 \times 10^{12} \\
3.00 \times 10^{13}\end{array}$ \\
\hline
\end{tabular}

\section{Discussion and Conclusion}

No attempt to use TART to rigorously fit the temperature dependence data for PMB has yet been reported, so as a first step we decided that this should be attempted.

The fitting was done on a computer by varying the parameters until satisfactory agreement between model and experiment was obtained. The results of this analysis are presented in Figs. 1 to 3 and Table 2. The equations used to model these temperatures dependencies are $[2,9,21]$

$$
\begin{aligned}
\frac{1}{T_{1}}= & \sum_{j=\mathrm{A}, \mathrm{B}, \mathrm{C}}\left\{C _ { \mathrm { AE } } ^ { j } \left[\frac{\tau_{\mathrm{c} j}}{1+\left(\omega_{\mathrm{t} j}-\omega_{0}\right)^{2} \tau_{\mathrm{c} j}^{2}}\right.\right. \\
& +\frac{\tau_{\mathrm{c} j}}{1+\left(\omega_{\mathrm{t} j}+\omega_{0}\right)^{2} \tau_{\mathrm{c} j}^{2}}+\frac{4 \tau_{\mathrm{c} j}}{1+\left(\omega_{\mathrm{t} j}-2 \omega_{0}\right)^{2} \tau_{\mathrm{c} j}^{2}}
\end{aligned}
$$

$$
\begin{aligned}
& \left.+\frac{4 \tau_{\mathrm{c} j}}{1+\left(\omega_{\mathrm{t} j}+2 \omega_{0}\right)^{2} \tau_{\mathrm{c} j}^{2}}\right] \\
& \left.+C_{\mathrm{EE}}^{j}\left[\frac{\tau_{\mathrm{c} j}}{1+\omega_{0}^{2} \tau_{\mathrm{c} j}^{2}}+\frac{4 \tau_{\mathrm{c} j}}{1+4 \omega_{0}^{2} \tau_{\mathrm{c} j}^{2}}\right]\right\},
\end{aligned}
$$

where $\tau_{\mathrm{c} j}$ and $\omega_{\mathrm{t} j}$ are given by

$$
\begin{aligned}
\frac{1}{\tau_{\mathrm{c} j}}= & \frac{1}{\tau_{0}^{j}} \exp \left\{-E_{\mathrm{a}}{ }^{j} / R T\right\} \\
& +\sum_{i} \frac{1}{\tau_{\mathrm{t} i}^{j}} \exp \left\{-E_{0 i}^{j} / R T\right\},
\end{aligned}
$$

and

$$
\omega_{\mathrm{t} j}=\frac{\sum_{i} \omega_{\mathrm{t} i}^{j} \exp \left\{-E_{0 i}^{j} / R T\right\}}{\sum_{i} \exp \left\{-E_{0 i}^{j} / R T\right\}},
$$

respectively, where $\tau_{0}^{j}$ and $\tau_{t i}^{j}$ are constants, the $E_{0 i}^{j}$ are the energy differences between the ground and 
$i^{\text {th }}$ excited torsional states and the $\omega_{\mathrm{t} i}^{j}$ are the tunneling splittings of the $i^{\text {th }}$ torsional state. The sum over $j$ is necessary because there are three nonequivalent methyl groups in the sample which we label, A, B and C, with methyl group A having the smallest tunneling splitting and $C$ the largest. The effective dipolar constants $C_{\mathrm{AE}}^{j}$ and $C_{\mathrm{EE}}^{j}$, within TART, are not easy to determine accurately because of their dependence on parameters such as $\delta^{2}$, the effective relaxation efficiency factor and the contribution from inter methyl group interactions. However, since these factors are expected to be of the order of 1 and 0.1 , respectively [8], it was not difficult to get a reliable estimate for them. Fine tuning was then done by adjusting the parameters interactively until the best fit between the model and the experiment was obtained.

For each non-equivalent methyl group, the first four spectral densities in (4) behave similarly as do the last two. Thus, in the discussion that follows and in Fig. 2 and 3 we consider each of these groups of spectral densities as a unit for each non-equivalent methyl group. This leaves us with only six groups of spectral densities instead of eighteen individual ones.

The modelling of published $T_{1}$ temperature dependencies studied at 10,25 and $30 \mathrm{MHz}$ [13] shows very good agreement between theory and experiment. The biggest discrepancy is near $30 \mathrm{~K}$ for 25 and $30 \mathrm{MHz}$ and is less than a factor of 2, Figure 1 . It should be noted that at temperatures around $30 \mathrm{~K}$ all of the spectral densities for methyl groups B and $\mathrm{C}$ contribute about equally to the relaxation. Furthermore, the excited torsional states begin to play a role in this temperature domain since higher order terms in $\tau_{\mathrm{c} j}$ and $\omega_{\mathrm{t} j}$ start to become important (see (5) and (6)). Quite clearly the TART modelling works very well at these temperatures and frequencies and any discrepancies may be attributed to uncertainty about the structural parameters. This apparent fit is very misleading, however, as it will be shown below.

The $40 \mathrm{MHz}$ temperature dependence $[15,16]$ also agrees quite well with the TART modelling except that the calculated curve shows a small indication of a Zeeman-tunneling resonance at about $40 \mathrm{~K}$ which is not seen in the experimental data. This should correspond to a small nonexponentiality in the recovery function, however; the indication is that it was not resolved in the relaxation rate analysis.
The $T_{1}$ recovery functions at $60 \mathrm{MHz}[15,16]$ show a strong nonexponentiality in the temperature region 25 to $35 \mathrm{~K}$. $T_{1}$ values were obtained from these recovery functions both from the initial slope and by decomposing the recovery functions into two exponential decays and calculating the corresponding time constants. The initial slope $T_{1}$ value and $T_{1 L}$ are shown in Figure 3.

The fitting obtained for the $60 \mathrm{MHz}$ data is qualitatively satisfactory although quantitatively there are significant discrepancies. The fit to the long component, which we obtained by removing the resonant spectral density from the equation for $T_{1}^{-1}$, is good in the region where the recovery functions have been decomposed into a long and a short component. However, when we consider the initial slope values we get satisfactory agreement between the experimental and theoretical curve (which includes the resonant spectral density) only near the resonant peak. For temperature above the resonant peak the disagreement between the model and the experiment may be the result of the nonexponentiality of the recovery functions. It is possible that the reported experimental $T_{1}$ values correspond to a weighted average of the two time constants $[15,16]$. At lower temperatures. this discrepancy is always significant.

It remains to estimate the accuracy of the fitted parameters, Table 2. According to the TART theory the fitted constants should be [8]

$$
\begin{aligned}
& C_{\mathrm{AE}}^{j}=\frac{9}{40} d^{2} p_{j}\left(1+X_{1}^{j}\right) \delta_{j}^{2}, \quad \text { and } \\
& C_{\mathrm{EE}}^{j}=\frac{9}{20} d^{2} p_{j} X_{2}^{j} \delta_{j}^{2},
\end{aligned}
$$

where $d^{2}=1.79 \times 10^{10} \mathrm{~s}^{-2}$ is the dipolar constant for a proton-proton interaction within the methyl group, $p_{j}$ the fraction of equivalent protons $\left(p_{\mathrm{A}}=\right.$ $\left.p_{\mathrm{B}}=6 / 16, p_{\mathrm{C}}=3 / 16\right), \delta_{j}^{2}$ the relaxation efficiency factor, typically $\delta^{2} \sim 0.6$ to 0.8 , and $X_{1}^{j}$ and $X_{2}^{j}$ are the contributions from inter-molecular dipolar interactions: $0<X_{1}^{j}, X_{2}^{j} \ll 1$. With $\delta^{2}=0.77$ and $X_{1}=$ $X_{2}=0.1$ all the values presented in Table 2 are within a factor of 2 of the values predicted by TART. It is hard to estimate the correlation times $\tau_{0}^{j}$, and $\tau_{\mathrm{t} i}^{j}$. The values quoted agree reasonably well with values given in the literature $[8,9,14]$. The activation energies $E_{\mathrm{a}}^{j}$ agree very well with earlier results $[11,15,16]$. 
Tunneling splittings for ground torsional levels were used to estimate the barrier heights for the different non-equivalent groups $\left(V_{3}^{\mathrm{A}}=2.7 \mathrm{kcal} / \mathrm{mole}\right.$, $V_{3}^{\mathrm{B}}=1.25 \mathrm{kcal} / \mathrm{mole}$ and $\left.V_{3}^{\mathrm{C}}=0.78 \mathrm{kcal} / \mathrm{mole}\right)$. These values were then used to calculate the higher order tunneling splittings from a Mathieu equation, leading to satisfactory agreement with the data quoted in the last column of Table 2.

The next phase of our research was to use the parameters obtained from this fit of temperature dependences to explain the measured $T_{1}$ dispersions. The results are shown in Figs. 4, 5, and 6. In the resonant region, between about 40 and $65 \mathrm{MHz}$, the $T_{1}$ recovery function is characterized by two relaxation times, $T_{1 L}$ and $T_{1 S}$. Since no such resonant region is observed at lower frequencies, this resonance corresponds to the fulfillment of the condition $2 \omega_{0}=\omega_{\mathrm{t}}^{B}$. Consequently, in this frequency range we have tried to model the frequency dependence of $T_{1 S}^{-1}$ using $J\left(2 \omega_{0}-\omega_{\mathrm{t}}^{B}\right)$, while the other spectral densities were used to represent $T_{1 L}^{-1}$. Below $40 \mathrm{MHz}$, which corresponds to the "off-resonance" region, all of the spectral densities, including $J\left(2 \omega_{0}-\omega_{t}^{B}\right)$ were used to model the data.

Using this procedure we find a very reasonable, although not complete agreement between the experimental results and the theoretical curves for the frequency dependencies of the long component of the relaxation data (Figures 4, 5,6). This is not the case for $T_{1 S}^{-1}$. However, since the resonant peak has been detected in the temperature dependence of $T_{1}$ at $60 \mathrm{MHz}$ and $32 \mathrm{~K}$, the fit to the frequency dependence at $32 \mathrm{~K}$ is still quite good (Figure 5); with the same parameter set theoretical curves were generated at $25 \mathrm{~K}$ and $40 \mathrm{~K}$ which are far from satisfactory (Figures 4,6 ). We suspect that this behavior is the result of fitting the resonant peak for the temperature dependences at $60 \mathrm{MHz}$. We have tried also to readjust some of the parameters which govern the spectral density $J\left(2 \omega_{0}-\omega_{\mathrm{t}}^{B}\right)$ to get better fits to frequency dependencies at 25 and $40 \mathrm{~K}$ without spoiling the fit to the temperature dependencies, however, this attempt has failed. No parameter set within TART can provide satisfactory theoretical curves for all three frequency dependencies of $T_{1 S}^{-1}$ as well as for the temperature dependence of $T_{1}$. This is clear evidence that the semiclassical theory does not describe correctly the behavior of the system at or near Zeeman-tunneling resonant peaks, although it is able to predict the frequency at which the maximum occurs. Consequently, we are proposing an alternate way of dealing with these resonant peaks [10].

It should be possible to calculate $T_{1 S}^{-1}$ near a $2 \omega_{0}$ $=\omega_{\mathrm{t}}$ resonance from an equation of the form

$T_{1 S}^{-1}=\frac{4 \pi}{h^{2}}\left|\left\langle E \frac{1}{2} \frac{1}{2}\left|\mathscr{H}_{\text {dip }}\right| A \frac{3}{2}-\frac{3}{2}\right\rangle\right|^{2} \delta\left(E_{\mathrm{i}}-E_{\mathrm{f}}\right)$,

where the ket $\left|A \frac{3}{2}-\frac{3}{2}\right\rangle$ represents the symmetric $\mathrm{CH}_{3}$ torsional ground state with $I=\frac{3}{2}$ and $m=-\frac{3}{2}$, $\left\langle E \frac{1}{2} \frac{1}{2}\right|$ represents the antisymmetric $\mathrm{CH}_{3}$ torsional ground state with $I=\frac{1}{2}$ and $E=\frac{1}{2}, \mathscr{H}_{\text {dip }}$ is the dipolar Hamiltonian, $E_{\mathrm{i}}$ the initial energy and $E_{\mathrm{f}}$ the final energy in the transition. We will approximate the delta function in (8) by the inverse of the tunneling line width $\Gamma_{\mathrm{c}}^{-1}$. Since our sample is a powder we have taken the powder average to get

$\overline{T_{1 S}^{-1}}=\frac{6}{16} \frac{27}{20} \frac{\gamma^{4} \hbar^{2}}{r^{6}} \frac{1}{2 \pi} \Gamma_{\mathrm{c}}^{-1}=1.40 \times 10^{9} \mathrm{~s}^{-1} \Gamma_{\mathrm{c}}^{-1}$,

where $\gamma$ is the gyromagnetic ratio and $r$, the inter proton distance, was taken to be $1.79 \AA$. The factor of $6 / 16$ is necessary since we are dealing with only 6 of the 16 protons per unit cell. Since in practice a $T_{1 S}^{-1}$ is necessary to determine $\Gamma_{\mathrm{c}}$ we will proceed to calculate $\Gamma_{\mathrm{c}}$ from (9) and compare these values with the full width at half maximum values, $\Gamma_{\mathrm{ex}}$, determined from the experimental frequency dependences. When this is done we find that, at $40 \mathrm{~K}, \Gamma_{\mathrm{c}}$ equals $37.8 \mathrm{MHz}$ compared with $48 \mathrm{MHz}$ for $\Gamma_{\text {ex }}$ while at $32 \mathrm{~K}$ we get 35.9 and $12 \mathrm{MHz}$ for $\Gamma_{\mathrm{c}}$ and $\Gamma_{\text {ex }}$, respectively. At $25 \mathrm{~K}$ the data do not allow an unambiguous determination of $T_{1 S}^{-1}$ at $2 \omega_{0}=\omega_{\mathrm{t}}$ so that the calculation of $\Gamma_{\mathrm{c}}$ at $25 \mathrm{~K}$ cannot be done.

On the basis of these results, we propose that the TART model cannot properly explain spin-lattice relaxation when the Zeeman and tunneling energy levels are near a resonant matching. It was suggested that the reason for this failure is that near resonance $T_{\mathrm{ZT}}$ becomes so short that, on the time scale of $T_{\mathrm{ZT}}$, the tunneling system can no longer be considered strongly coupled with the lattice. Furthermore, it is likely that the resonant transfer of order between the Zeeman and tunneling systems is caused by a microscopic mechanism which is different from the ones that determine the other relaxation processes in the material. This resonant transfer should, therefore, have a different correlation time the temperature dependence of which is not known. 
We also investigated the Zeeman tunneling resonance using saturation experiments which were performed at a temperature and frequency such that twice the Zeeman splitting, $\hbar \omega_{0}$, was equal to the tunneling splitting, $\hbar \omega_{\mathrm{t}}$. The condition $\omega_{\mathrm{t}}=\omega_{0}$ should yield similar results. The appropriate frequencies were chosen using the $T_{1}$ frequency dependences mentioned above.

In Table 1, the results of the four pulse saturation experiment are compared with the calculated values for the magnetization following each pulse. The calculations are based on the assumption that the populations equilibrate if the matching is resonant. This can be called an order transfer three bath model $[10,18]$. According to this model the magnetization following the $n^{\text {th }}$ pulse (when the $\omega_{\mathrm{t}}=2 \omega_{0}$ condition is fulfilled) should be

$$
M_{n}=\left(\frac{2}{3}\right)^{n+1}(1-y)\left(1+\frac{y}{2}\right)^{n-1} M_{0},
$$

where $M_{0}$ is the equilibrium magnetization, and $y=\exp \left(-\tau / T_{1 S}\right)$. This equation can be used as long as both $\tau$ and $T_{1 S}$ are much smaller than $T_{1 L}$. In PMB, however, the longest $\tau$ used was of the order of $T_{1 L}$. As a result, the magnetization growth due to the Zeeman-lattice flow was not negligible. It was assumed, however, that both processes are independent, and that the Zeeman-lattice relaxation (which is concurrent with Zeeman-tunneling transfer) changes only the apparent semi-equilibrium magnetization value observed at the end of the order-transfer process. By extrapolating this value to shorter times and assuming an exponential growth of both components, we obtained the equation

$$
\begin{aligned}
M_{n}= & M_{0}\left\{R\left(\frac{2}{3}\right)^{n+1}(1-y)\left(1+\frac{y}{2}\right)^{n-1}\right. \\
& \left.+\left[1-R\left(\frac{2}{3}\right)^{n+1}\right]\left(1-e^{-\tau / T_{1 L}}\right)\right\},
\end{aligned}
$$

where $R$ is the fraction of the methyl groups which fulfill the $\omega_{\mathrm{t}}=2 \omega_{0}$ condition. We used $R=0.4$ [20].
Using this model we find good qualitative agreement between the experimental values and the calculated ones, but quantitatively they differ by as much as $30 \%$.

The results of the saturation experiments do prove, however, that in a time of the order of $T_{1 S}$ the system supplying order to the Zeeman reservoir becomes saturated. This is precisely what we would expect if the order were supplied by the torsional (tunneling) reservoir since it has a finite specific heat. The lattice, on the other hand, has at these temperatures an essentially infinite specific heat and can therefore never become saturated with this technique. This evidence along with the frequency dependence of the short relaxation time can only be explained by interpreting $T_{1 S}$ as the time constant which characterizes the transfer of order between torsional and Zeeman systems and does not involve the lattice. In other words, it is a measure of the strength of the Zeeman-torsion coupling; i.e., $T_{1 S} \equiv T_{\mathrm{ZT}}$.

In conclusion, it was shown that the frequency dependence of the Zeeman-torsion coupling time cannot be explained in terms of the semiclassical perturbation theory. This demonstrates that the processes determining the tunneling linewidth are different from the processes determining the spinlattice relaxation. The former is determined by symmetry conserving torsional ground state to first excited state transitions (where both states are in the same potential well) while the latter is caused by transitions above the hindering barrier. For this reason the problem of tunneling assisted spin-lattice relaxation is complicated. The frequency dependence of the Zeeman-torsional coupling does, however, provide very accurate information on the $\mathrm{CH}_{3}$ tunneling frequencies and on the tunneling linewidth, while the saturation experiments yield information about the specific heat of the different reservoirs involved in the observed relaxation. These kinds of data, unlike the temperature dependence of the relaxation times at fixed frequencies, provide a better insigth into the effect of rotational tunneling in solids. 
[1] J. H. Freed, J. Chem. Phys. 43, 1710 (1965).

[2] J. Haupt, Z. Naturforsch. 26 a, 1578 (1971).

[3] M. Punkkinen and S. Clough, J. Phys. C7, 3403 (1979).

[4] R. A. Wind, S. Emid, D. J. Ligthelm, J. F. J. M. Pourquié, and J. Smidt, Bull. Mag. Res. 6,71 (1984).

[5] S. Clough, J. Phys. C9, 1553 (1976).

[6] W. Muller-Warmuth and R. Schuler, J. Chem. Phys. 69, 2382 (1978).

[7] D. J. Ligthelm, R. A. Wind, and J. Smidt, Physica $100 \mathrm{~B}, 175$ (1980).

[8] D. J. Ligthelm, Thesis, University of Technology, Delft 1981 .

[9] J. F. J. M. Pourquié, Thesis, University of Technology, Delft 1979.

[10] B. S. Bharaj and M. M. Pintar, Phys. Rev. Lett. 52, 1986 (1984).

[11] P. S. Allen and A. Cowking, J. Chem. Phys. 49, 789 (1968)
[12] P. S. Allen and S. Clough, Phys. Rev. Letters 22, 1351 (1969).

[13] K. Morimoto, J. Phys. Soc. Japan 39, 1413 (1975).

[14] B. Gabryś and L. Van Gerven, Chem. Phys. Lett. 82, 260 (1981).

[15] B. Gabryś, Thesis, Leuven 1982.

[16] B. Gabryś and L. Van Gerven, J. Phys. C: Solid State Phys., 18, 1241 (1985).

[17] K. Morimoto, J. Phys. Soc. Japan 50, 2404 (1981).

[18] K. R. Sridharan, W. T. Sobol, and M. M. Pintar, J. Chem. Phys. 82, 4886 (1985).

[19] W. G. Clark and J. A. McNeil, Rev. Sci. Instrum. 44, 844 (1973).

[20] S. Clough and A. J. Horsewill, Chem. Phys. Lett. 82, 264 (1981).

[21] W. Muller-Warmuth, H.-H. Duprée, and M. Prager, Z. Naturforsch, 39 a, 66 (1984). 